\title{
Bringing Patient Care Back to the Bedside: Collaborative Clinical and Academic Leadership
}

Mohamed Panju MD, Leslie Martin BMSc, MD, FRCPC, Marianne Talman MD, Juan Guzman MD, MSc, FRCPC, Khalid Azzam MD, MBBS, DABIM, FACP, FRCPC, CPE

\begin{abstract}
About the Authors:
Mohamed Panju is Associate Professor, Division of General Internal Medicine, Department of Medicine, McMaster University. Leslie Martin is Assistant Professor, Division of General Internal Medicine, Department of Medicine, McMaster University. Marianne Talman is Associate Professor, Department of Medicine, McMaster University. Juan Guzman is Associate Professor, Division of General Internal Medicine, Department of Medicine. McMaster University. Khalid Azzam is Associate Professor of Medicine, Division of General Internal Medicine, McMaster University.

Correspondence to Mohamed Panju: mohamed.panju@medportal.ca

Submitted: January 12, 2018. Accepted: February 20, 2018. Published: November 9, 2018. DOI: 10.22374/cjgim.v13i4.269
\end{abstract}

Academic tertiary care centres face several challenges in providing excellent patient care and medical education. These centres provide service to high volumes of medically complex patients with multiple needs. In addition, there is demand for an efficient patient flow to accommodate newly admitted patients and discharge stable patients to their homes with appropriate care plans. A large portion of the medical care for patients in academic hospitals is provided by medical learners (medical students, residents, and fellows). Accommodating learners has its own set of challenges. This includes providing a safe learning environment, exposure to a variety of medical cases, and appropriate supervision. The Royal College of Physicians and Surgeons of Canada is moving towards Competency Based Medical Education (CBME) which is an educational paradigm gaining attention and uptake international. ${ }^{1,2} \mathrm{CBME}$ requires direct observation of learners by supervising faculty. To ensure excellence in both clinical care and medical education, there needs to be strong collaboration between both hospital and educational leadership.

The internal medicine (IM) service at the Hamilton General Hospital has taken a collaborative approach to help integrate achieving the educational objectives with the provision of excellent patient care and efficient patient flow. In order to achieve this, a novel model for reviewing new patients each morning was established.

As an academic IM training site, our inpatient medicine service has at least six medical teams on service at any time with six different attending physicians. Four of the teams have medical learners while the other teams are staffed by a physician and nurse practitioner. One of the teaching teams provides IM consultation and admission when necessary for patients referred from the Emergency Department between 8 am and $4 \mathrm{pm}$ during weekdays. The other teams accept patients in the morning, who have been admitted overnight by the medical house-staff on call. Overnight medical admissions are completed by a minimum of four residents (junior and senior) plus one to two medical students. The patients are reviewed with the senior resident overnight. To ensure that morning handover, including reviewing patients with the attending physicians and the teams, is effective and efficient and to improve patient flow with timely patient management decision, several interventions were introduced.

\section{A Novel Model of Bedside Patient Presentations}

Each evening the junior residents and medical students are assigned to a specific team to admit patients. The junior learner assigned, whenever possible, is a member of that teaching team. The overnight resident and/or student are able to handover $(8-10 \mathrm{am})$ to a single dedicated attending physician and their team of learners. Each morning there are, on average, between 2-6 patients admitted to each of the teaching teams. The junior learner presents the patients to the attending physician and the medical team at the bedside in the presence of the patient, family, and when possible the bedside nurse. These changes have the potential for several clinical and educational benefits. 


\section{Optimizing Clinical Care}

Clinically, patients are seen earlier with the case presentation at the bedside with the entire team. Patient care and treatment decisions are made with the team of residents who are able to provide assistance in executing the plan of care. This includes performing tasks such as calling to expedite a diagnostic test, communication with a pharmacy or a consultant, etc. The entire house-staff also becomes familiar with the new patients admitted to the team. This helps with providing care throughout the day as the residents are able to deal with issues arising. This also helps with safe handover at the end of the day. When the bedside nurse is involved in this process, there is a shared mental model that also includes the nursing staff. The ability to provide efficient and timely decisions on day one of admission has the potential to shorten hospital length of stay. With regards to patient experience, the patients and their families become aware of the structure of a medical teaching team. They are introduced to the team members including junior resident who will manage day-to-day issues, the senior resident and the supervising physician. Patients are also involved in their care from first day of admission and are updated with the next step in their care.

\section{Educational Benefits of Bedside Patient Presentations}

"He who studies medicine without books sails an uncharted sea, but he who studies medicine without patients does not go to sea at all" - William Osler

Team rounding on newly admitted patients has several educational benefits. The medical trainees are able to learn new concepts in context of an acute medical case. Each new patient presentation at the bedside functions like a miniature morning report. This allows for learning to be moved from the classroom back to the bedside. In addition, the supervising physician is able to provide immediate feedback to the resident who admitted the patient. By presenting the patient at the bedside the supervising physician is able to comment directly on the management plan. The supervising physician is also able to directly observe physical exam skills and explore the learner's cognitive thought process regarding investigations and the workup of the patient. This level of direct observation and immediate feedback will help to facilitate workplace-based assessment and decisions regarding entrustment in the era of CBME. ${ }^{3}$

\section{Setting the Stage}

To ensure there was appropriate infrastructure for morning bedside handover, the clinical and educational leadership in our hospital took a collaborative approach. The clinical and educational leaders needed to ensure support and buy-in from the supervising physician group. The benefits of this approach were explained to involved physicians. In addition, informal presentations on how to conduct morning bedside rounding were delivered. The on-call schedule for learners was updated to ensure that there were specific learners dedicated to the teaching teams each evening. The clinical physician leadership also had to rally support for the Emergency Department and ward nursing leadership and staff. The large group of learners spending time at the bedside of newly admitted patients could appear to be disruptive to nursing workflow. To avoid this undesired outcome, the potential positive patient-care outcomes were explained in advance to secure support at all levels. The method of team rounding on newly admitted patients has become part of the hospital culture for the IM service.

\section{Next Steps}

Moving forward, we will need to assess our model of assessing new patients as a team at the bedside. From a clinical point of view, we will need to assess if we are able to shorten hospital stays, ensure that new admissions are safer and evaluate if we are improving the patient experience and satisfaction. From an educational aspect, we will be able to evaluate if we are able to provide better evaluations and feedback for our learners. This may help as we transition to CBME. Overall it has been an exciting process to bring patient care back to the bedside.

\section{References}

1. 1. Carraccio C, Englander R, Van Melle E, et al. Advancing CompetencyBased Medical Education: A Charter for Clinician-Educators. Acad Med 2016;91(5):645-49. doi:10.1097/acm.0000000000001048

2. 2. Frank JR, Mungroo R, Ahmad Y, et al. Toward a definition of competencybased education in medicine: a systematic review of published definitions. Med Teach 2010;32. doi:10.3109/0142159x.2010.500898

3. 3. Ten Cate O, Hart D, Ankel F, et al. Entrustment Decision Making in Clinical Training. Acad Med 2016;91(2):191-98. doi:10.1097/ acm.0000000000001044 